\title{
Synchronization of groups of coupled oscillators with sparse connections
}

\author{
Zhigang Zheng ${ }^{1(a)}$, Xiaoqin Feng ${ }^{1},{\text { Bin } \text { Ao }^{2} \text { and Michael C. Cross }}^{1,3}$ \\ ${ }^{1}$ Department of Physics and the Beijing-Hong Kong-Singapore Joint Centre for Nonlinear and Complex Studies, \\ Beijing Normal University - Beijing 100875, China \\ ${ }^{2}$ Center for Nonlinear Studies and Department of Physics, Hong Kong Baptist University - Hong Kong, China \\ ${ }^{3}$ Department of Physics, California Institute of Technology - Pasadena 91125, CA, USA
}

received 21 July 2009; accepted in final form 31 August 2009

published online 24 September 2009

PACS 05.45.Xt - Synchronization; coupled oscillators

PACS 89.75.-k- Complex systems

\begin{abstract}
Synchronization of groups of coupled oscillators with sparse connections are explored. It is found that different topologies of intergroup couplings may lead to different synchronizability. In the strong-coupling limit, an analytical treatment and criterion is proposed to judge the synchronization between communities of oscillators, and an optimal connection scheme for the group synchronization is given. By varying the intergroup and intragroup coupling strengths, different synchronous phases, i.e., the unsynchronized state, intragroup synchronization, intergroup synchronization, and global synchronization are revealed. The present discussions and results can be applied to study the pattern formation and synchronization of coupled spatiotemporal systems.
\end{abstract}

Copyright (c) EPLA, 2009

Synchronization, as a universal cooperative behavior and a fundamental mechanism of self-organizations in nature, has been extensively studied in recent years [1-6]. The synchronization of spatiotemporal systems have attracted much attention, and numerous interesting bifurcations and on-off intermittent behaviors are found [7]. The recent focus on complex networks has given much insight on the relation between the topological properties of coupled oscillators and collective dynamics [8]. The structural properties of a complex network may significantly affect the dynamical processes. It has been found that networks with a homogeneous distribution of connectivity are more synchronizable than heterogeneous ones (e.g., scale-free networks) [9]. Synchronization can also be effectively improved on weighted networks [10].

Networks with community structures arise ubiquitously in social and biological sciences [11]. On such networks, elements in a society tend to form groups according to their social characteristics, and connections among elements in one group are dense while connections among elements in different groups are typically sparse. It is of potential interest to investigate the synchronization between two groups (we call it group synchronization, $\mathrm{GrS}$ )

\footnotetext{
(a) E-mail: zgzheng@bnu .edu.cn
}

when only a few intergroup connections are considered. Even for a given number of intergroup links, there can be many different ways of connections between two groups. The synchronization dynamics between two communities may be completely different for different network topologies. Therefore it is important to study this issue and give theoretical approaches or criteria for the emergence of GrS for a given coupled community network. This should be helpful in understanding the dynamical processes and their implications in relating to biological or social issues about community networks.

In this letter, we explore the synchronization of communities of coupled oscillators with sparse connections. It is found that different topologies of intergroup couplings may lead to different synchronizability. In the strong-coupling limit, we propose an analytical treatment and criterion to judge the synchronization between communities of oscillators, and an optimal connection scheme for the group synchronization is given. By varying the intergroup and intragroup coupling strengths, different synchronous phases, i.e., the unsynchronized state, intragroup synchronization, intergroup synchronization, and global synchronization are revealed. Our exploration reveals the significant role of network topology in governing the global dynamics. The present discussions can be applied 
to study the pattern formation and synchronization of coupled spatiotemporal systems.

The dynamics on a given network can be written as

$$
\dot{\vec{X}}=\vec{F}(\vec{X})+\Gamma \otimes C \vec{X},
$$

where $\vec{X}=\left(\vec{x}_{1}, \vec{x}_{2}, \ldots, \vec{x}_{N}\right)$ denotes dynamical variables, $\vec{F}=\left(\vec{f}_{1}, \vec{f}_{2}, \ldots, \vec{f}_{N}\right)$ represents the nonlinear functions governing the dynamics of oscillators, and $N$ is the number of nodes, and $\Gamma: R^{m} \rightarrow R^{m}$ describes the coupling scheme among the nodes. $C$ is the adjacent matrix representing the links among elements in a network, and $\sum_{j=1}^{N} C_{i j}=0$, with the largest eigenvalue $\lambda_{1}^{C}=0\left(\lambda_{1} \geqslant \lambda_{2} \geqslant \cdots \geqslant \lambda_{N}\right)$.

For simplicity, we consider a network composed of two identical groups, with each group being $N$ chaotic oscillators (labelled as $i=1,2, \ldots, N$ ) with nearest-neighbor coupling $\varepsilon$. Between two groups, there are $n_{l}<N$ connections with the intergroup coupling strength $r$, which are labelled as $L_{1}, L_{2}, \ldots, L_{n_{l}}$. A network with the this structure is marked as $2 * N\left(L_{1}, L_{2}, \ldots, L_{n_{l}}\right)$. We use indices $i$ and $i^{\prime}$ to represent the corresponding nodes in two groups.

In the following discussions we apply the typical threedimensional Rossler oscillator as our nodes: $\dot{x}=-(y+$ $z), \dot{y}=x+a y, \dot{z}=b+z(x-c)$, and each node is linearly coupled to others through the $y$ component. Throughout this letter we set $a=0.15, b=0.4, c=8.5$. The results we observed for the Rossler oscillators in this letter can be naturally generalized to other cases of node dynamics.

We first numerically study the dynamics for the case of $N=8$ and $n_{l}=2$. Dynamical equations eqs. (1) are numerically integrated in terms of Runge-Kutta augorithm with the time step $\Delta t=0.001$. The synchronization between two oscillators $i$ and $j$ can be measured by introducing the distance of two states as $\quad d_{i j}=\lim _{T \rightarrow \infty}\left(T^{-1} \int_{0}^{T}\left\|\vec{x}_{i}(t)-\vec{x}_{j}(t)\right\| \mathrm{d} t\right)$, where $\|\cdot\|$ can be the Euclidean distance. GrS is achieved if $d_{i i^{\prime}}=0$ but $d_{i j} \neq 0$ for all $i, j, i^{\prime}$. Intragroup synchronization occurs when $d_{i j}=0$ and $d_{i^{\prime} j^{\prime}}=0$ but $d_{i i^{\prime}} \neq 0$. If for all sites in two groups satisfy $d_{i j}=0$, $d_{i^{\prime} j^{\prime}}=0$, and $d_{i i^{\prime}}=0$, global synchronization can be achieved.

The synchronous dynamics for a network $2 * 8(2,7)$ in fig. 1(a) are presented in fig. 1(b). We keep the coupling ratio $r / \varepsilon=0.8$ and increase both $r$ and $\varepsilon$. It can be found that GrS takes place prior to intragroup synchronization. For a different network topology $2 * 8(2,4)$ (see fig. $1(\mathrm{c})$ ), the synchronization dynamics become completely different, as shown in fig. 1(d), where $r / \varepsilon=1$. It can be found that GrS does not occur prior to complete synchronization, in fact they are reached at the same critical coupling strength. The contrasting results reveal that different network structures may lead to different synchronization phenomena, and the structural properties have a great impact on the synchronous dynamics. Therefore an important question naturally arises: what kind of networks can support the group synchronization? Furthermore, which structure can lead to an optimized GrS? It is thus very
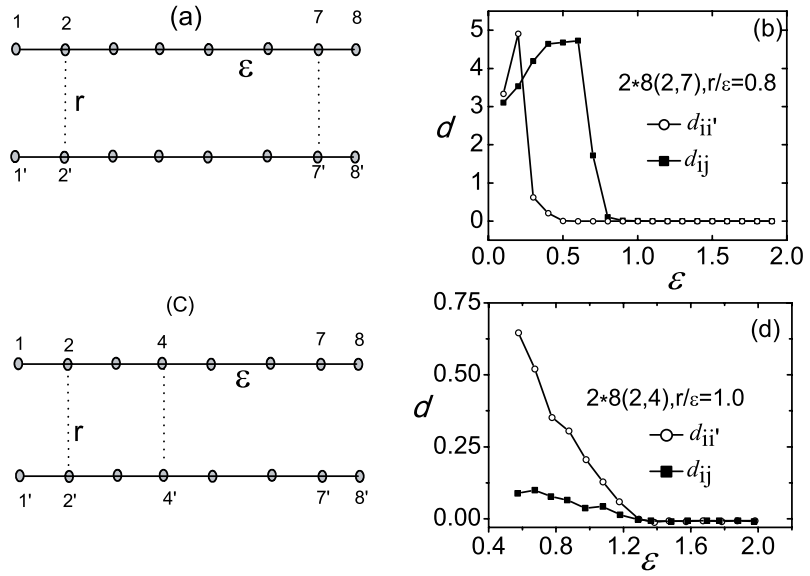

Fig. 1: (a) The topological graph of the network $2 * 8(2,7)$. (b) The relation between the average distance and the coupling strength $\epsilon$ for $r / \varepsilon=0.8$. (c) The topological graph of networks with two coupled chains $2 * 8(2,4)$. (d) The relation between the average distance and the coupling strength on the network at $r / \varepsilon=1.0$.

important to ravel the condition for the emergence of group synchronization.

The stability of complete synchronization (CS) can be well solved by applying the master stability function scheme [12]. CS is determined by the stability of the transversal subsystem. For two sparcely coupled communities, GrS can be considered as the CS between two groups, but it is difficult to give an eminent analytical expression because each group is a high-dimensional dynamical system. Recently, the partial synchronization (PaS) of coupled oscillators was explored theoretically and numerically [13], and analytical criterion for the emergence of $\mathrm{PaS}$ has been proposed. Obviously, the topology of two coupled groups satisfies the mirror symmetry, hence the treatment of PaS may be well applied to theoretical analysis of $\mathrm{GrS}$.

The mirror symmetry of the network topology implies the invariance of the adjacent matrix $C$ under the similarity transformation: $F_{2 N} C F_{2 N}^{-1}=C$, where $F_{2 N}$ is a counteridentity matrix satisfying $F_{i, 2 N+1-i}=1$, $i=1,2, \ldots, 2 N$ and other elements are all 0 . Furthermore the adjacent matrix can be block diagonalized by constructing a similarity transformation $S$ :

$$
M=S^{-1} C S=\left(\begin{array}{cc}
A & 0 \\
0 & B
\end{array}\right),
$$

where

$$
S=\left(\begin{array}{cc}
I_{N} & F_{N} \\
I_{N} & -F_{N}
\end{array}\right),
$$

and $I_{N}$ is the $N$-order identity matrix. By introducing new variables $\left(\vec{G}_{l}, \vec{G}_{r}\right)=S\left(\overrightarrow{X, X^{\prime}}\right)$, where $\vec{G}_{l}=\vec{X}+$ $\vec{X}^{\prime}, \vec{G}_{r}=\vec{X}-\vec{X}^{\prime}$, and $\vec{G}_{l}=\left(\vec{g}_{l}^{1}, \vec{g}_{l}^{2}, \ldots, \vec{g}_{l}^{N}\right)$ and $\vec{G}_{r}=$ $\left(\vec{g}_{r}^{1}, \vec{g}_{r}^{2}, \ldots, \vec{g}_{r}^{N}\right)$. The similarity matrix $S$ does not change the eigenvalues of the matrix $C$. It only redistributes them 
(a)

(b)

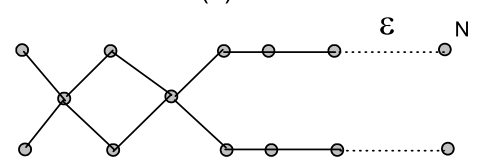

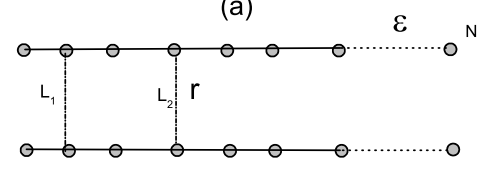

(c)

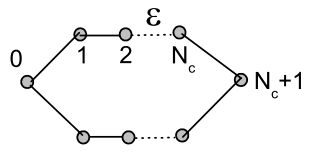

(d)

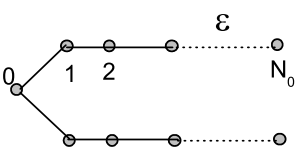

Fig. 2: The whole system is divided into $N_{c}$-close-block and $N_{o}$-open-block in the condition of infinite connection strength between groups.

to two blocks $A$ and $B$. In fact, $\vec{G}_{l}$ denotes the dynamics of the globally synchronous (GIS) manifold, and $\vec{G}_{r}$ denotes the dynamics on the GrS manifold. Then the system can be divided into two parts. It is obvious that the GrS state is the solution of eq. (1). It is important to study the stability of the GrS state. The dynamics near the GrS state can be written as

$$
\begin{gathered}
\delta \dot{\overrightarrow{G_{l}}}=\left[D \vec{F}\left(\overrightarrow{G_{l}}\right)+\Gamma \otimes A\right] \delta \overrightarrow{G_{l}}, \\
\delta \dot{\overrightarrow{G_{r}}}=\left[D \vec{F}\left(\overrightarrow{G_{r}}\right)+\Gamma \otimes B\right] \delta \overrightarrow{G_{r}},
\end{gathered}
$$

where

$$
\left[\Gamma \otimes A\left(\delta \vec{G}_{l}\right)\right]^{i}=\varepsilon \Gamma\left(\delta G_{l}^{i+1}+\delta G_{l}^{i-1}-2 \delta G_{l}^{i}\right) .
$$

For oscillators without intergroup couplings,

$$
\left[\Gamma \otimes B\left(\delta \overrightarrow{G_{r}}\right)\right]^{i}=\varepsilon \Gamma\left(\delta G_{r}^{i+1}+\delta G_{r}^{i-1}-2 \delta G_{r}^{i}\right),
$$

$i=1,2, \ldots, N$. For oscillators coupled with intergroup links:

$$
\left[\Gamma \otimes B\left(\delta \overrightarrow{G_{r}}\right)\right]^{j}=\varepsilon \Gamma\left(\delta G_{r}^{j+1}+\delta G_{r}^{j-1}\right)-2(\varepsilon+r) \Gamma \delta G_{r}^{j}
$$

According to the criterion for the emergence of $\mathrm{PaS}$ [13], the criterion for the emergence of $\mathrm{GrS}$ is

$$
\lambda_{2}^{l}>\lambda_{1}^{r}
$$

where $\lambda_{2}^{l}$ is the second largest eigenvalue of $A$, and $\lambda_{1}^{r}$ is the largest eigenvalue of $B$.

Let us further approach analytical results for the criterion of GrS. In the strong intergroup coupling limit, i.e., $r \rightarrow \infty$, as shown in fig. 2(a), two directly coupled oscillators can be regarded as a common node of two groups (see fig. 2(b)). Therefore two coupled chains can be divided into several parts with two types of fundamental topologies: the ring block with $2\left(N_{c}+1\right)$ units (see fig. 2(c)) and the chain block with $2 N_{o}+1$ oscillators (see fig. 2(d)). The possibility of GrS depends crucially on the synchronizability of these fundamental blocks. Note that synchronizability for both fundamental structures can be theoretically analyzed, hence one may obtain analytical results by combining the criterion (7) for GrS .

In the strong-coupling limit, for the dynamics $\vec{G}_{l}=\vec{X}+$ $\vec{X}^{\prime}$ on the GlS manifold, the eigenvalues of the connection matrix can be worked out as

$$
\lambda_{q}^{l}=-4 \varepsilon \sin ^{2}(q / 2), \quad q=m \pi / N,
$$

where $m=0,1,2, \ldots, N-1$.

For the dynamics $\vec{G}_{r}=\vec{X}-\vec{X}^{\prime}$ on the GrS manifold in the regime $r \rightarrow \infty$, the eigenvalues of the connection matrix for a ring block are given by

$$
\lambda_{q}^{r}=-4 \varepsilon \sin ^{2}(q / 2), \quad q=m \pi /\left(N_{c}+1\right) .
$$

Here $m=1,2, \ldots, N_{c}$.

For the case of chain blocks, the eigenvalues of the connection matrix can also be analytically worked out as

$$
\lambda_{q}^{r}=-4 \varepsilon \sin ^{2}(q / 2), \quad q=(2 m-1) \pi /\left(2 N_{o}+1\right),
$$

where $m=1,2, \ldots, N_{o}$.

The second largest eigenvalue for the GlS manifold is $\lambda_{2}^{l}=-4 \varepsilon \sin ^{2}(\pi / 2 N)$. For the GrS manifold, the largest eigenvalue for the transversal manifold is $\lambda_{1}^{r}=-4 \varepsilon \sin ^{2}\left(\pi / 2\left(N_{c}+1\right)\right)$ for the ring block. For the chain structure, one has $\lambda_{1}^{r}=-4 \varepsilon \sin ^{2}\left(\pi /\left(2 N_{o}+1\right)\right)$. By applying the criterion ( 7$)$ for $\mathrm{GrS}$, one can naturally give rise to the following conclusions:

- For both types of blocks, the larger the size of a block is, the larger $\lambda_{1}^{r}$ is, and the more difficult for $\lambda_{2}^{l}>\lambda_{1}^{r}$. Therefore GrS becomes difficult if the size of one of the blocks in the network becomes very large.

- If there exists a chain block with $N_{o} \geqslant N / 2$, i.e., its size exceeds one half of the network size, one has $\lambda_{2}^{l}<\lambda_{1}^{r}$, i.e., GrS will not be observed.

- A chain block with the size $n$ has the same eigenvalue $\lambda_{1}^{r}$ and the same synchronization ability as a ring block with size $2 n$.

- The minimum number of intergroup links is $n_{l}=2$, i.e., GrS cannot be achieved when there is only one intergroup link. For the case of $n_{l}=2$, the optimal way for GrS is the topology that includes form two chains with the size $n=(N-2) / 4$ and one loop block with the size $(N-2) / 2$.

In terms of the above theoretical arguments, it is not difficult to understand the synchronization phenomena in the aforementioned networks $2 * 8(2,7)$ and $2 * 8(2,4)$. For the network $2 * 8(2,7)$, it is possible to achieve $\mathrm{GrS}$ when $r / \varepsilon>0.42$. While for the structure $2 * 8(2,4)$, there is one chain with $N_{o}=4=N / 2$, and $\lambda_{2}^{l}\left[=-4 \varepsilon \sin ^{2}(\pi / 16)\right]$ $<\lambda_{1}^{r}\left[=-4 \varepsilon \sin ^{2}(\pi / 18)\right]$. So GrS cannot be reached. Note 


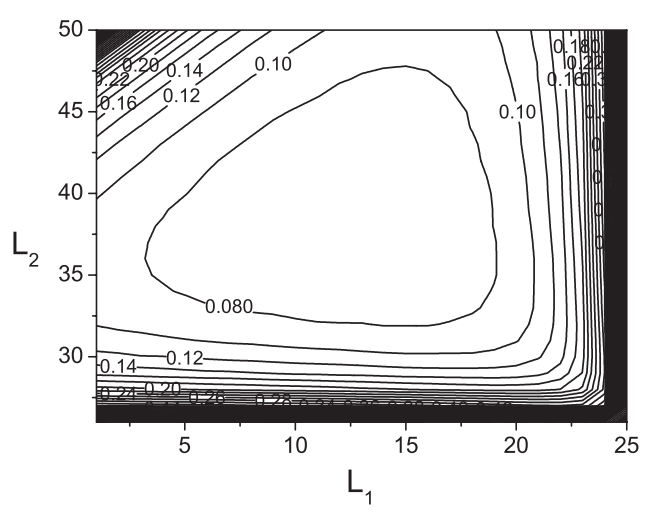

Fig. 3: The critical intergroup coupling $r_{c}$ for GrS varying with different ways of links $\left(L_{1}, L_{2}\right)$ for a large network $2 * 50\left(L_{1}, L_{2}\right)$. The contour line is for critical coupling strength $r_{c}$ for GrS.

that the above conclusions are made in the strong intergroup coupling limit, numerical simulations indicate that they are still valid for finite couplings. Numerical experiments also well verify the conclusion that a chain block with size $n$ possesses the same synchronization ability with the a ring block with size $2 n$. These discussions implies that the largest block is the crucial for the emergence of GrS, and it determines the range of the critical $r_{c}$ for GrS in this network.

For the same number of intergroup links, the way of links may strongly affect the emergence of GrS. For a large network, the more symmetrical the intergroup connections are, the easier the groups get synchronized and the smaller the critical $r_{c}$ is. We numerically computed $r_{c}$ varying with different ways of links $\left(L_{1}, L_{2}\right)$ for a network with $N=50$ in each lattice and $n_{l}=2$ intergroup connections, as shown in fig. 3. It can be found that in the middle regime the critical coupling $r_{c}$ is lower, indicating that links separating into sub-networks (chains or loops) with homogeneous synchronizability optimize the GrS.

Apart from the GrS state, one can find other forms of synchrony. One may draw a phase diagram on the $(\varepsilon, r)$-plane to describe transitions among different forms of synchrony for a given group network. In terms of the necessary condition for GrS, the size of the largest chain block should be less than half size of a group, $n_{o}<N / 2$. Moreover, the ratio between intergroup connections and intragroup couplings should be large enough, i.e., coupling strength among groups should be relatively larger. Based on the criterion of GrS that $\lambda_{2}^{l}>\lambda_{1}^{r}$, the phase diagram can be divided into four parts: unsynchronized state (US), group synchronization state (GrS), intragroup synchronization state(IS) (elements in one group synchronize to each other), and complete synchronization state(CS). In fig. 4(a), we draw the $\varepsilon-r$ phase diagram for the network $2 * 10(3,8)$, and four dynamical phases are labelled. In
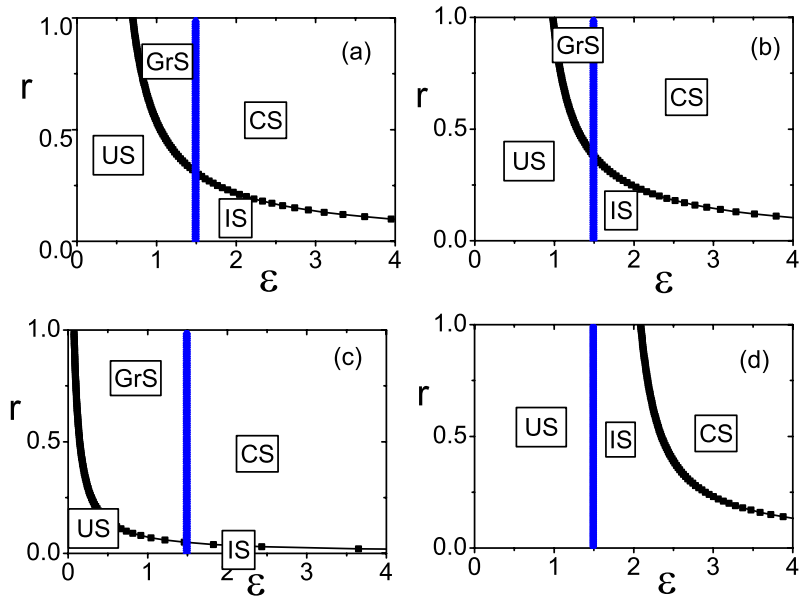

Fig. 4: (Colour on-line) Dynamics Phase diagrams for different community networks $2 * 10\left(L_{1}, L_{2}\right)$. (a) $2 * 10(3,8)$; (b) $2 * 10(2,7) ;$ (c) $n_{l}=N$, i.e., all nodes are connected; (d) $2 * 10(6,8)$.

order to give a comaprison we also give the phase diagram for other topologies in figs. 4(b), (c), and (d). If there are only two connections, the topology $2 * 10(2,7)$ in 4 (b) possesses a smaller parameter regime for $\mathrm{GrS}$ as compared with the optimal topology $2 * 10(3,8)$ in $4(a)$. If all pairs between groups are connected, i.e., $n_{l}=N$, one has the largest the parameter area for GrS (see fig. 4(c)). For the topology $2 * 10(6,8)$, there is a chain block with a size larger than $N / 2=5$, so GrS can never occur and the area for $\mathrm{GrS}$ never exists.

In conclusion, in this letter we study the synchronization of communities of coupled oscillators with sparse links. Our results concern how the network synchronization ability depends on the sparce links. It is found that different topologies of intergroup couplings may lead to different synchronizability. In the strong-coupling limit an analytical treatment and criterion is proposed to judge the emergence of GrS. Phase diagrams of different synchronous phases are revealed. The key elements for $\mathrm{GrS}$ is the size of the open blocks and close blocks. Recent works have indicated that group-structure networks are important for biological networks such as metabolic networks and protein interaction graphs [14]. The present studies reveal the intrinsic collective bifurcation of coupled dynamical systems prior to the emergence of global synchronization. We hope that the present studies can shed light on some current problems such as synchronization dynamics, pattern dynamics and network dynamics.

This work is supported in part by the National Natural Science Foundation of China, the National Basic Research Program of China, the Foundation for Doctoral Training from MOE and the HSCC of Beijing Normal University. 


\section{REFERENCES}

[1] Pikovsky A., Rosenblum M. and Kurths J., Synchronization: A Universal Comcept in Nonlinear Sciences (Cambridge University Press, Cambridge) 2001 and references therein.

[2] Zheng Z. G., Wang X. G. and Cross M. C., Phys. Rev. E, 65 (2002) 056211.

[3] Rosenblum M. G., Pikovsky A. S. and Kurths J., Phys. Rev. Lett., 76 (1996) 1804.

[4] Zheng Z. G., Hu G. and Hu B., Phys. Rev. Lett., 81 (1998) 5318.

[5] Hu G., Zhang Y., Cerdeira H. A. and Chen S. G., Phys. Rev. Lett., 85 (2000) 3377.

[6] Chen Z., Hu K., Stanley H. E., Novak V. and Ivanov P. CH., Phys. Rev. E, 73 (2006) 031915; Xu L., Chen Z., Hu K., Stanley H. E. and Ivanov P. Ch., Phys. Rev. E, 73 (2006) 065201.
[7] Zhan M., Zheng Z. G., Hu G. and Peng X. H., Phys. Rev. E, 62 (2000) 3552.

[8] Albert R. and Barabasi A. L., Rev. Mod. Phys., 74 (2002) 47; Barabasi A. L. and Albert R., Science, 286 (1999) 509; Watts D. J. and Strogatz S. H., Nature (London), 393 (1998) 440.

[9] Nishikawa T., Motter A. E., Lai Y. C. and HoppenSteadt F. C., Phys. Rev. Lett., 91 (2003) 014101.

[10] Motter A. E., Zhou C. S. and Kurths J., Europhys. Lett., 69 (2005) 334.

[11] Park K., Lai Y. C., Gupte S. and Kim J. W., Chaos, 16 (2006) 015105.

[12] Pecora Louis M., Phys. Rev. E, 58 (1998) 347.

[13] Ao B. and Zheng Z. G., Europhys. Lett., 74 (2006) 229; Ao B., Ma X. J., Li Y. Y. and Zheng Z. G., Chin. Phys. Lett., 23 (2006) 786.

[14] Pogromsky A., Physica D, 172 (2002) 65. 